\title{
El sufrimiento en la catástrofe. Orientaciones para una agenda de investigación en perspectiva relacional
}

LUIS CAMPOS MEDINA

> Instituto de la Vivienda, Universidad de Chile, Santiago, Chile. Iuiscampos@uchile.fau

Universidad de Valparaíso

Facultad de Arquitectura

Revista Márgenes

Espacio Arte Sociedad

El sufrimiento en la catástrofe. Orientaciones para una

agenda de investigación en perspectiva relacional

Diciembre 2014 Vol. $11 \mathrm{~N}^{\circ} 15$

Páginas 49 a 56

ISSN elec. 0719-4463

ISSN imp. 0718-4034

Recepción: Junio 2014

Aceptación: Noviembre 2014

\section{RESUMEN}

En este artículo propongo una perspectiva relacional para la consideración del sufrimiento en la catástrofe. El propósito es programático, por lo cual describo la perspectiva conceptual, explicito supuestos e hipótesis y propongo una vía metodológica específica acorde a la conceptualización. La perspectiva conceptual propuesta supone tomar distancia respecto de la habitual consideración mentalista del sufrimiento, enfatizando las dimensiones socio-cultural y socio-espacial involucradas. Dicha perspectiva conceptual es interdisciplinar, ya que propongo articulaciones entre planteamientos provenientes de la geografía humana contemporánea, la sociología, la antropología y la historia. En el plano metodológico, la perspectiva relacional implica prestar atención a las prácticas, materialidades e interdependencias ligadas al sufrimiento y la catástrofe, para lo cual sugiero un abordaje etnográfico, acompañado de trabajo en archivos, entrevistas en profundidad, recorridos comentados y producción de cartografías. Concluyo indicando que destacar la relevancia del sufrimiento en la catástrofe implica intervenir sobre el debate público, intentando posicionar a quienes sufren como sujetos dignos de consideración por parte de los poderes públicos y del conjunto de la sociedad civil.

PALABRAS CLAVE

sufrimiento, catástrofe, perspectiva relacional, articulación socio-espacial

\section{The suffering in the disaster. Guidelines for an agenda of research on relational perspective}

ABSTRACT

In this article I propose a relational perspective to consider suffering in the catastrophe. The purpose is programmatic. I describe the conceptual perspective; I make explicit the assumptions and the hypotheses; and I propose a specific methodological way in link with this conceptualization. The conceptual perspective involves taking distance from the usual mentalist consideration of suffering, emphasizing the socio-cultural and sociospatial dimensions. This conceptual approach is interdisciplinary and I generate links between approaches from contemporary human geography, sociology, anthropology and history. At the methodological level, I claim for an attentive approach to practices, materiality and interdependencies, linking to suffering and catastrophe. To do this the ethnographic approach is especially pertinent, accompanied by work in archives, interviews, commented tours and production of maps. I conclude indicating that give importance to suffering in the catastrophe implies intervene on the public debate, trying to give relevance to sufferers as worthy subject of consideration by public authorities and the whole of civil society.

KEYWORDS

suffering, catastrophe, relational perspective, socio-spatial articulation

\section{La souffrance dans la catastrophe. Lignes directrices pour un program- me de recherche en perspective relationnel RÉSUMÉ}

Dans cet article, nous proposons une perspective relationnelle pour considérer la souffrance dans la catastrophe. Le but est programmatique, c éest pour cela que nous décrivons le point de vue conceptuel, les supposés et les hypothèses et nous proposons un chemin méthodologique spécifique conforme à cette conceptualisation. Le point de vue conceptuel proposé suppose prendre distance de l'habituel examen mentaliste de la souffrance, en mettant l'accent sur les dimensions socioculturelles et sociospatiale impliquées. Cette perspec- 
tive conceptuelle est interdisciplinaire, car nous proposons I' articulation entre des approches de la géographie humaine contemporaine, la sociologie, l'anthropologie et l' histoire. Sur le plan méthodologique, la perspective relationnelle implique une attention aux pratiques, aux matériaux et aux interdépendances liées à la souffrance et à la catastrophe, raison pour laquelle nous proposons une approche ethnographique, accompagnée du travail dans les archives, d’entretiens en profondeur, parcours commenté et production de cartographies. Nous concluons en suggérant que la mise en évidence de la pertinence de la souffrance dans la catastrophe implique intervenir sur le débat public, en essayent de placer ceux qui souffrent comme sujets dignes de considération par les pouvoirs publics et l'ensemble de la société civile.

MOTS CLÉS

souffrance, catastrophe, perspective relationnelle, articulation

\section{INTRODUCCIÓN}

En un sugerente texto publicado en 1993, T. A. Lavell planteaba que el abordaje de los desastres por parte de las ciencias sociales latinoamericanas no daba cuenta de una dimensión central de los mismos: su carácter eminentemente social. Usualmente ponderados en relación a la cantidad de pérdidas humanas que producen o a las pérdidas económicas que generan, la adjetivación mayoritariamente empleada seguía siendo la de "natural", oscureciendo así la causalidad social involucrada.

Esta situación se ha ido modificando de modo progresivo, como lo atestigua una importante variedad de publicaciones recientes (Cid, et. al., 2012; Aliste y Pérez, 2013; González-Muzzio, 2013; Musset, 2010; Ugarte y Salgado, 2014), siendo en la actualidad casi imperativo hablar de desastres socio-naturales. Independientemente del indiscutible valor de estas investigaciones y propuestas comprensivas, la conceptualización ofrecida en la mayoría de los abordajes revisados no toma en cuenta -o lo hace de modo periférico- una dimensión crucial, como es la relativa a las emociones. Nuevamente se impone la dificultad de pensar y representar lo afectivo e integrarlo al análisis de la conducta social (Favret-Saada, 1994).

En una revisión somera encontramos que dichos abordajes llevan la discusión hacia aspectos centrales, como son: las percepciones e interpretaciones subjetivas de los riesgos (Cid, et. al., 2012); la relevancia de las permanencias históricas y la memoria colectiva en los procesos de reconstrucción (Aliste y Pérez, 2013); avanzadas conceptualizaciones de capital social, como la ofrecida por González-Muzzio que lo entiende como "activo multinivel" (González-Muzzio, 2013); la justicia espacial (Musset, 2010); o bien la centralidad de las formas de acción colectiva y constitución de sujetos (Ugarte y Salgado, 2014). No obstante, las emociones casi no son consideradas en la problematización, a excepción de la referencia transversal, pero incipiente, al sufrimiento.

En efecto, la ocurrencia de una catástrofe es siempre ligada, en el relato analítico, a una experiencia de sufrimiento, ya sea por parte de la población concernida, las edificaciones maltrechas o el territorio de ocurrencia. Estas diversas entidades "sufren" los desastres socio-naturales, sólo que la noción de sufrimiento movilizada equivale a la idea de haber experimentado los embates del desastre y no hay consideración del papel que ese sufrimiento juega en la producción misma del desastre o la catástrofe. Tres consecuencias analíticas se desprenden de lo anterior. Primero, que el sufrimiento de personas, edificaciones y territorios es sobre todo un recurso narrativo empleado por los autores para dar cuenta de los hechos que son objeto de su atención. Segundo, que el sufrimiento es una ocurrencia que afecta a diversas entidades de modo aislado: sufre el territorio, el edificio y la persona. Tercero, que a pesar de esto último, el sufrimiento surge siempre en contextos de los cuales es imposible aislarlo. A partir de este tercer elemento es que a continuación propondremos una concepción diferente del sufrimiento, la que puede otorgar un punto de partida distinto para reflexionar y analizar la dimensión "social" de los desastres y catástrofes socio-naturales, esta vez desde una perspectiva que puede denominarse "relacional" y que implica ponderar de forma distinta el valor de aspectos sociales, culturales, temporales y espaciales.

\section{POR UN ABORDAJE RELACIONAL DEL SUFRIMIENTO}

La palabra "sufrimiento" nos remite a las ideas de dolor, padecimiento y pena, vale decir, a un tipo de experiencia humana de afección y malestar cuyo origen puede ser tanto de orden físico como moral. Pero la palabra sufrimiento también Ileva consigo la referencia a la modalidad en que se vivencia ese dolor, padecimiento o pena. El sufrimiento es, asimismo, la paciencia, conformidad y tolerancia con que se sufre algo.

De la referencia anterior se desprende que el sufrimiento se encuentra asociado semánticamente, e incluso vivencialmente, a otras nociones tales como las de dolor, o de padecimiento, y que ese sufrimiento adopta una forma específica que es observable por otros. En el abordaje que las ciencias sociales han hecho del sufrimiento el empeño principal ha estado puesto en mostrar que éste posee una dimensión sociocultural, vale decir, que tanto la experiencia singular de sufrimiento, como la forma expresiva que éste adopta, se encuentran moldeadas socioculturalmente.

El libro “La cultura del dolor", de D. Morris (1991) es un buen ejemplo. En él el autor señala que el dolor no es nunca un asunto puramente médico, sino que un fenómeno en el que participa de modo decisivo la cultura, aun cuando su acción pase inadvertida ante nuestros ojos. Adoptando un enfoque histórico, Morris intenta mostrarnos cómo el dolor que sentimos actualmente no es el que sentían nuestros antepasados y que la asimilación actual entre dolor e impulso eléctrico nervioso es el resultado de la influencia decisiva de la perspectiva médica sobre la cultura contemporánea. El empeño de Morris es el de mostrarnos que el dolor es histórico, ya que se ve continuamente reconfigurado por un tiempo, lugar y cultura. Para dicho autor, como para otros (Damasio 2013; Moscoso 2011; Zombory-Nagy y Frandon 1994) el sufrimiento no es una entidad que se localiza en el cerebro humano, sino que en la intersección de cuerpos, mentes y culturas. Es posible plantear, en consecuencia un primer argumento para esta agenda de investigación: si el sufrimiento se produce en la intersección de cuerpos, mentes y cul- 
turas, entonces, esta intersección debe manifestarse espacialmente o, dicho con otras palabras, debe ocurrir en algún sitio.

El sentido común indica que el sufrimiento es algo personal, propio, que cada uno vive individualmente. Pero del argumento anterior se desprende que resulta plausible adoptar una perspectiva distinta: el sufrimiento no es una entidad que se encuentra restringida a la conciencia y/o a la mente individual, sino que, por el contrario, es de orden socio-espacial e inter-subjetivo. Siguiendo al geógrafo Nigel Thrift, cabe entender que el sufrimiento, al igual que cualquier otra entidad del mundo, se encuentra espacialmente distribuida (Thrift, 2006) y que esa distribución espacial puede ser aprehendida intelectualmente y caracterizada desde un punto de vista socio-geográfico.

Si el punto anterior es viable, cabe añadirle un corolario: si el sufrimiento es una entidad espacialmente distribuida, él se encuentra inseparablemente ligado al tiempo y al movimiento, tal como plantea Thrift a través de su noción de timespace (2006). Es pertinente, entonces, proponer que el sufrimiento puede ser concebido como una modalidad de interacción dinámica entre un conjunto de seres animados que se afectan recíprocamente (Thrift, 2008:8385). Así las cosas, el sufrimiento sería una realidad emergente, surgida de las relaciones mutuas y siempre cambiantes que articulan a un conjunto de elementos, humanos, pero también no-humanos (como objetos, materialidades, animales, entre otros), los cuales se pueden encontrar en relación de cercanía física, o bien distantes en el espacio-tiempo.

\section{POR UN ABORDAJE SOCIOCULTURAL DEL SUFRIMIENTO}

Haciendo eco de un conjunto de tradiciones científicas que buscan alejarse de la concepción del sufrimiento que le entiende como una condición mental-subjetiva-interior, lo que se busca relevar aquí es que el sufrimiento puede ser concebido en términos de sus articulaciones socio-espaciales, más que como estados mentales subjetivos interiorizados (Davidson, Bondi y Smith, 2007). Entre dichas tradiciones, una fundamental es la histórica, encarnada en el proyecto del fundador de la Escuela de los Anales, Lucien Febvre.

En uno de los textos que componen el libro "Combats pour I'histoire" de 1952, el historiador francés se preguntaba por la relevancia e interés del estudio de las emociones para la disciplina histórica. Su respuesta era afirmativa y categórica. En el despliegue de su argumento, Febvre echaba mano de un texto antiguo de la Enciclopedia Francesa para mostrar cómo, ya en el siglo XVIII, la investigación psicológica de punta proponía comprender la emoción no como una pura reacción automática del organismo a un estímulo exterior, pasajera y mecánica, sino que entenderla como un fenómeno más complejo, que llega a constituir un sistema de incitaciones interindividuales.

La emoción, para Febvre, implica relaciones entre seres humanos $y$, en consecuencia, relaciones colectivas, por lo que cabe considerarla como una institución. Sin embargo, parece claro que su concepción de la emoción no es -o no puramente- la de un estado mental subjetivo. De hecho, en el sentido recién señalado de institución, su propuesta conecta directamente con la visión que Marcel Mauss (1921) desarrolla en el texto "L'expresion obligatoire des sentiments” (“La expresión obligatoria de los sentimientos”).

Efectivamente, fiel a la perspectiva durkheimiana, Mauss consideraba que la expresión de los sentimientos no era una simple "ma- nifestación", sino que un verdadero lenguaje, una "simbólica". Las emociones no sólo se manifiestan, sino que se manifiestan-a-otros y esta condición es la que supone que se constituyan en signos que pueden ser, y de hecho son, interpretados y entendidos por otros (Mauss, 1921). Vale decir, las emociones son eminentemente relacionales y suponen la existencia de un colectivo en el cual se expresan y moldean. Más aún, la emoción no es concebible fuera de ese colectivo y de esa forma relacional, así como tampoco es concebible la existencia de un lenguaje privado (Wittgenstein, 2008).

En una perspectiva similar se sitúa la propuesta contemporánea del antropólogo francés David Le Breton. El autor, en su “Antropología del dolor", propone entender el sufrimiento como la significación otorgada por un individuo a una determinada experiencia que percibe como dolorosa (Le Breton, 1999). Dicha significación se construye en el marco de una determinada cultura, de lo que se desprende que, primero, el sufrimiento no es nunca una entidad sólo de orden biológico y, segundo, que su mediación cultural es condición para que se presente a la conciencia de quien lo experimenta (Le Breton, 1999; Thumala, 2003).

Nuevamente aquí vemos que la emoción, particularmente el dolor, no constituye un estado interno y, en consecuencia, que su comprensión no puede ser desligada de las circunstancias en que se produce, las que incluyen una serie de elementos que van desde el lenguaje, hasta aspectos culturales y lógicas sociales locales (Rosaldo, M., 1980; Wierzbicka \& Jamrozik, 1988; Rosaldo, R., 1989; Geertz, 2002; Boltanski, 2007).

La perspectiva sociocultural de la emoción encuentra un punto de expresión especialmente interesante entre los llamados teóricos culturalistas de los sentimientos, quienes defienden un enfoque de las emociones esencialmente semiótico -las ven como instrumentos de significación y prácticas constructivas a través de los cuales aquellas adquieren forma, sentido y curso público (Geertz, 2002:198). Desde esta perspectiva, las emociones articulan un entramado de relaciones que se dan, no sólo en el plano ritual, sino que también en marcos informales de la vida cotidiana. Se trata muchas veces de prácticas menos circunscritas que los ritos u otras formas de acción organizada. Sin embargo, igualmente implican el uso de un lenguaje específico (para el cual muchas veces no existe traducción sencilla ni directa), la asignación y construcción de un sentido social, y una lógica que trasciende la situación específica y el tiempo en que se manifiesta dicha práctica.

Sólo que para estos autores la consideración de aspectos lingüísticos y situacionales como meros efectos o síntomas de una emoción, entendida como un estado neuronal o fisiológico, no tiene mayor sentido. El delineamiento de lo que es una emoción supone una consideración atenta de distintos elementos que ponen en relación a individuos, y otros tipos de entidades, en una particular producción significativa: palabras, imágenes, gestos, marcas corporales y terminologías, las historias, los ritos, costumbres, arengas, melodías y conversaciones no son meros vehículos [...] Son el lugar y el mecanismo de la cosa misma (Geertz, 2002:198).

Esa particular atención a entidades diversas, que no son meros vehículos de la emoción, refuerza la idea de que el sufrimiento se encuentra en la intersección de cuerpos, mentes y culturas. Esa intersección puede ser concebida como un punto de encuentro circunstancial cuya extensión en el tiempo (Porée, 1997) y en el espacio es variable, pero, al igual que un cántico, un abrazo o una vela en un pequeño altar, siempre posee una expresión socio-espacial. 


\section{POR UN ABORDAJE SOCIO-ESPACIAL DEL}

\section{SUFRIMIENTO}

Similar ha sido la deriva de los estudios de geografía agrupados bajo la etiqueta del emotional turn, en los que se pretende integrar a la comprensión geográfica el rol jugado por las emociones en la vida cotidiana de la gente. De acuerdo al texto señero "Emotional geographies" (Davidson, Bondi y Smith, 2007), las geografías emocionales se ven cruzadas por tres focos de interrogación principales: en primer lugar, la pregunta por dónde están localizadas las emociones; en segundo lugar, la pregunta por cómo las emociones se producen en relaciones entre las personas y sus entornos y; en tercer lugar, la pregunta por las formas y modos de representar las emociones. Tales interrogantes tienen importantes implicancias. Primero, implican concebir las emociones como entidades que se encuentran localizadas en algún sitio y que no son etéreas, sino que responden al principio de distribución referido anteriormente. Segundo, implican concebirlas como entidades relacionales y no como esencias o sustancias independientes de la vinculación intersubjetiva. La tercera interrogante, siendo de orden epistemológico, implica reflexionar sobre los modos de abordar y conocer un tipo de entidades que resultan elusivas a la representación.

Los mismos autores (Davidson, Bondi y Smith, 2007) recuerdan que las emociones recién surgen doscientos años atrás, alrededor de 1820 y que antes se hablaba de otras cosas: sentimientos, pasiones o afecciones. Esto no quiere decir que la gente entonces no sintiera pena o alegría, sino que las nombraba de otra manera y, muy probablemente, las experimentaba o vivenciaba de un modo que hoy nos resultaría extraño. Con esto los autores ponen de manifiesto tanto la importancia de un abordaje histórico de la emoción, como la relevancia de las maneras de nombrar, pues ellas participan de la experiencia.

Este último elemento no es menor, pues evidencia un nuevo punto de convergencia con el tratamiento dado a las emociones por parte de los teóricos culturalistas de los sentimientos mencionados en el apartado anterior. Pero sobre todo, porque indica la variedad contextual que posee la experiencia humana de la emoción, y porque pone de manifiesto que en el modelamiento de la experiencia emotiva participan una serie de factores, dentro de los cuales el lenguaje tiene un rol prominente. Por cierto que esto no carece de antecedentes. El mismo Fevbre (1952), planteaba que los instrumentos para llevar a cabo una historia de la sensibilidad y la vida afectiva eran principalmente tres, entre los cuales la importancia dada al lenguaje resulta evidente: el vocabulario de una época (a través de su análisis lingüístico y filológico), la iconografía artística y la literatura de determinado período histórico.

Ahora bien, en la reflexión de las ciencias sociales sobre las emociones, frecuentemente se plantea que una emoción está ligada a su opuesto (Febvre, 1952; Moscoso, 2011; Mascareño, 2006). En el caso del sufrimiento, ese opuesto pareciera ser la felicidad. Partiendo de esta base, una breve mención a la reflexión en torno de la felicidad hecha por Ahmed (2011) puede resultar útil para entender la relevancia de un abordaje socio-espacial del sufrimiento.

En su artículo "Happy objetcs", Ahmed (2010) plantea que la etimología de la palabra inglesa "happiness" la conecta con la contingencia, puesto que en el inglés medio "hap" sugiere la noción de "chance", es decir, de oportunidad. Recurriendo a la reflexión hecha por la tradición del empiricismo inglés, en particular a la obra de John Locke, Ahmed plantea que para dicho autor la felici- dad es idiosincrática: nos hacen felices cosas diferentes y encontramos encantadoras cosas distintas. Por otra parte, desde una perspectiva fenomenológica, la felicidad puede ser descrita como “intencional”, en cuanto está siempre orientada hacia objetos, así como "afectiva", puesto que está en contacto con objetos. De esto se desprende que la felicidad es una orientación hacia los objetos con los que estamos en contacto (Ahmed, 2010:32, traducción personal).

La reflexión hecha por la autora en relación a los objetos es interesante, además, por cuanto plantea que éstos pueden devenir "felices" por asociación, vale decir, por el solo hecho de encontrarse cerca de un "objeto feliz" (“happy object”). La felicidad es, en esa medida, contagiosa, de lo que se deriva que los afectos, más que provenir desde el interior de un sujeto, se transmiten a través de los cuerpos, en una dinámica relacional e interactiva.

La felicidad, entonces, puede jugar un rol crucial en el proceso de dar forma a nuestra esfera próxima, el mundo que nos rodea como un mundo de cosas familiares (Ahmed, 2010:32, traducción personal). En esa misma dirección, aquellos objetos que mantenemos alejados de nosotros son reveladores de nuestro mundo: rechazando la cercanía de algunos objetos, definimos los lugares que sabemos que no queremos visitar, las cosas que no queremos poseer, tocar, probar, oír, sentir, ver, aquellas cosas que no queremos mantener al alcance (Ahmed, 2010:32, traducción personal).

Aplicar este razonamiento al caso del sufrimiento resulta bastante plausible. El sufrimiento también parece ligado a eventos que acontecen situacionalmente, es decir, que tienen la oportunidad de afectarnos en el marco de una situación determinada, la que se compone de una serie de entidades articuladas recíprocamente: objetos, materialidades, significados, etc. Junto con ello, el sufrimiento también se insinúa como contagioso: por ejemplo, estar frente a alguien que sufre puede hacernos sufrir; o, por ejemplo, las veces en que intentamos evitar aquellos objetos y situaciones que producen sufrimiento. En ese sentido, el sufrimiento aparece como la articulación de una esfera y de un entrelazamiento con el mundo. De modo que el sufrimiento podría ser descrito en base a la noción de awayness (Ahmed, 2010), dado que se trataría de una situación o articulación en la cual no es posible alejar de nosotros aquellas cosas que no nos gustan: debemos estar siempre con ellas, en contacto con ellas, padeciéndolas.

En ese sentido es que cabe concebir también de un modo relacional la catástrofe socio-natural: ella se sufre justamente porque implica una articulación circunstancial de objetos, materialidades, sujetos, corporalidades, etc., que invaden e intervienen de improviso aquella esfera próxima que hemos configurado a través de la constitución -cotidiana y permanente- de distancias espaciales y temporales con un conjunto de entidades indeseables. Infausta intervención del orden regular de las cosas producto de un evento específico.

\section{PROPUESTA CONCEPTUAL: MODALIDADES SOCIO-ESPACIALES DE ARTICULACIÓN DEL SUFRIMIENTO}

Vistos así, sufrimiento y catástrofe se encuentran espacialmente distribuidos. Pero ello no implica que se traten de entidades estáticas, fijas o inmóviles. Muy por el contrario, el hecho de que posean una espacialidad implica que se trata de entidades que se encuentran en movimiento y que son inseparables del tiempo (Thrift, 2006)1. 
Vistas así, una agenda de investigación que reconoce el carácter relacional del sufrimiento y la catástrofe requiere reconocer que ambos siempre se encuentran en proceso, vale decir, que siempre se despliegan en la forma de prácticas que articulan espacios, tiempos, materialidades y escalas, junto con normas, valores, modelos de relación y conducta, y roles (Revel, 2005:64).

Dicho con otras palabras, la observación del sufrimiento y la catástrofe supone prestar atención a articulaciones emergentes que no pueden ser predichas en función de alguna forma de determinismo estructural. Tales articulaciones son dinámicas, es decir se modifican a través del tiempo, y es en función de ellas que adquiere consistencia la experiencia social de los individuos involucrados en una forma de desastre.

Resulta plausible entonces proponer una noción teórica y operativa para el estudio del sufrimiento y la catástrofe: la idea de modalidades de articulación socio-espacial. Por ella se puede entender una configuración circunstancial de relaciones inter-subjetivas e inter-objetuales que se modifica a través del tiempo, dando forma, significado, consistencia y duración a la experiencia. Cada una de las entidades que conforma una determinada articulación socioespacial provee de sentido a la experiencia de sufrimiento y la pone en relación con los espacios, tiempos, historias y relaciones con las que dicha entidad se encuentra asociada.

En este sentido, la idea de modalidad de articulación socio-espacial constituye una herramienta operativa que facilita la observación empírica de la experiencia de la catástrofe y el sufrimiento y, al mismo tiempo, entrega un punto de partida teórico para entrar en diálogo con nociones fundamentales de la geografía, tales como territorio, lugar y paisaje. De esta forma, observando una modalidad de articulación se podrá identificar cuerpos, objetos, materialidades, individuos cercanos y lejanos, así como grupos de referencia y pertenencia que participan de ella. A su vez, cada una de esas entidades permitirá establecer conexiones con otros dominios de actividad y otros marcos de sentido, para así entretejer las eventuales formas territoriales de la catástrofe y el sufrimiento.

Por ejemplo, el tratamiento analítico de las modalidades de articulación socio-espacial del sufrimiento en un caso específico puede dar pie a establecer conexiones con la noción de lugar, en particular en las vertientes trabajadas como "altos lugares" (Bédard, 2002, 2002a), "pequeños altos lugares” (Musset, 2008) y “lugares móviles" (Jirón e Iturra, 2011), en la medida que se observe la incidencia específica de micro-prácticas espaciales a través de las cuales se va produciendo, de modo inevitable y muchas veces no intencional y una vinculación significativa con un determinado tipo de emplazamiento.

\section{HACIA UNA AGENDA DE INVESTIGACIÓN RELACIONAL PARA LA CATÁSTROFE Y EL SUFRIMIENTO}

Una agenda de investigación que pretenda instalar una consideración relacional de la catástrofe y el sufrimiento debe hacer frente, entonces, a dos concepciones persistentes en el tratamiento de ambas entidades. En primer lugar, respecto del sufrimiento, se debe enfrentar la concepción que le entiende como un padecimiento que experimentan los individuos en su fuero íntimo, como una sensación de dolor que involucra sólo la interioridad de quien la soporta. Esta es una visión que puede ser denominada mental e internalista del sufrimiento y que puede ser contrastada con la idea esbozada más arriba de que el sufrimiento puede ser concebido como una experiencia en la que se articulan cuerpos, mentes, culturas, tiempos y espacios. En segundo lugar, se debe hacer frente a la concepción que entiende la catástrofe como un evento que es "recibido" circunstancialmente por una entidad, generalmente humana, para pasar -siguiendo la concepción de espacio propuesta por Thrift (2006)-, a una concepción que la entiende como una producción contingente que: a. comporta diferentes escalas y duraciones de modo simultáneo, b. configura redes de afectación que se superponen e imbrican.

Llevar adelante esta agenda de investigación relacional supone movilizar una diversidad de enfoques metodológicos y técnicas, con la finalidad de ganar mayor sensibilidad empírica en un trabajo fuertemente inductivo. En efecto, no obstante la discusión que hemos desarrollado y la propuesta conceptual efectuada, el carácter "relacional" que hemos propuesto puede ser fácilmente expresable, pero sin duda es más difícil de observar y caracterizar empíricamente. Esto hace necesario desarrollar investigación en terreno de modo profuso e intenso, eludiendo las respuestas teóricas previas respecto de lo que es el objeto analítico de indagación (Lash 2009:177, citado en Back, 2007: 21). Esto es, evitar lo que en sociología S. Lash ha denominado el "a priori simbólico", cuyo efecto principal es disminuir nuestra capacidad de abordar las formas emergentes y los fenómenos incipientes, especialmente aquellos que se plantean de un modo contra-intuitivo como aquí hemos hecho. En coherencia con esta perspectiva "a posteriori", los conceptos y enfoques de los que partimos deben ser concebidos como "operadores analíticos iniciales", por cuanto establecen el punto de partida para la construcción del objeto analítico, pero se trata de una construcción provisoria que se irá modificando con en el transcurso de la investigación empírica².

El abordaje metodológico de tipo multi-métodos es pertinente, además, por las constataciones hechas en la literatura contemporánea, donde se muestra que el sufrimiento y el desastre, particularmente de tipo socio-ambiental, no son necesariamente experimentados de maneras unívocas, ni descritos de maneras claras y nítidas por parte de los involucrados. Más bien se trata de experiencias atravesada por la confusión y, muchas veces, por la negación (Auyero y Swistun, 2008:21).

En el caso del sufrimiento ambiental, Auyero y Swistun (2008:2425 ) indican que la literatura especializada describe una secuencia que va del descubrimiento del peligro ambiental, a la conciencia creciente sobre las consecuencias generadas por el factor tóxico, a la aparición de vecinos que se transforman en líderes y luego en militantes de las causas ambientales, y a un proceso de aprendizaje en el que las víctimas se vuelven hábiles receptores y usuarios del conocimiento científico y se transforman en agentes políticos frente a las autoridades. Sin embargo, dicha secuencia no es la que ellos encontraron en un su estudio empírico.

Es aquí que se vuelve necesario explicitar un supuesto ontológico a tener presente en la relación entre sufrimiento y catástrofe, cual es que, a mayor intensidad del sufrimiento, los individuos suelen evidenciar una mayor restricción de sus referencias y marcos espacio-temporales a nivel discursivo y práctico. Es decir, cuanto más sufren, más circunscrito el ámbito espacio-temporal al que aluden discursivamente y en el que se desenvuelven de modo práctico. En base a este supuesto se vuelve plausible considerar, a modo de hipótesis de trabajo básica, que las formas de articulación socio-espacial del sufrimiento serán más estables -menos circuns- 
tanciales- en la medida que haya una menor preponderancia de elementos discursivos y una mayor preponderancia de elementos no-discursivos.

Esto implica que las perspectivas que se avocan a la comprensión de la conciencia que tienen los individuos acerca del sufrimiento y al discurso que producen en relación a él son necesarias, pero insuficientes (Auyero y Swistun 2008; Back y Puwar 2012; Bourdieu 1999; Kleinman et al. 1997; Mol 2002; Wilkinson 2005) ${ }^{3}$. En efecto, incluso los enfoques mentales e internalistas reconocen que el sufrimiento es una entidad elusiva del discurso, esto es, que para los individuos resulta muchas veces difícil expresar verbalmente el sufrimiento que padecen (Riessman 2008). La propuesta es aquí mostrar que el método etnográfico posee la plasticidad y adaptabilidad que un abordaje multi-métodos requiere, ya que permite combinar la observación y la escucha, la perspectiva del observador y de los involucrados, así como dar relevancia de las materialidades, temporalidades y espacialidades en la experiencia del sufrimiento.

\section{ORIENTACIONES METODOLÓGICAS}

En consecuencia, intentar aprehender la espacialidad relacional del sufrimiento y la catástrofe requiere tomar distancia de las metodologías y técnicas que parten del supuesto de que se trata de una entidad mental e interna y que generalmente recurren exclusivamente a aproximaciones discursivas. Por el contrario, si el sufrimiento es concebido como: a. una experiencia que emerge en la relación o intersección del individuo con su entorno socio-cultural y material; b. una experiencia-en-proceso que se despliega en la forma de prácticas que articulan espacios, tiempos, materialidades, normas, valores, modelos de relación, roles, etc., resulta entonces necesaria una aproximación que posibilite observar tales relaciones, articulaciones y dinámicas. Vale decir, una aproximación que permita observar prácticas, así como reconocer el valor informativo de distintos tipos de materiales, incluyendo los discursivos, pero sin restringirse a ellos: que el sufrimiento sea una entidad elusiva del discurso, no implica que no haya discursos sobre el sufrimiento, ni discursos que lo moldeen o que contribuyan a darle forma. Implica que la aparición de esos discursos puede ser efímera, circunstancial y que producir un discurso sobre su propia experiencia de sufrimiento puede implicar un gran trabajo para los individuos.

De esta forma, si la modalidad de articulación socio-espacial constituye una herramienta operativa que facilita la observación empírica de la catástrofe y el sufrimiento, también puede ser concebida como un operador que permite identificar la situación a estudiar -la unidad de análisis-y, al mismo tiempo, modelar el enfoque etnográfico en una suerte de "acompañamiento situacional”, en el cual combinar la observación participante y no participante, la producción de discursos y narrativas, la perspectiva del observador y de los involucrados, así como incluir en el análisis a las materialidades, las temporalidades y las espacialidades de la experiencia del sufrimiento. En esta línea cabe destacar que existen variadas experiencias de investigación en las que se genera una versión particular del enfoque etnográfico (Jirón, 2010; Wacquant, 2006).

Además, cabe recordar que el enfoque aquí propuesto no es el del sufrimiento como experiencia individual, sino que en su condición relacional, interactiva y dinámica, lo que implica necesariamente un abordaje que complemente cuestiones discursivas y no discursivas. Pero que además se haga cargo de la incidencia de otros actores. Nuevamente aquí son relevantes los planteamientos de
Auyero y Swistun cuando subrayan que Quienes sufren no experimentan su situación como aislados Robinson Crusoes sino en contextos relacionales y discursivos específicos. Estos contextos le dan forma a las maneras en que los actores viven y entienden su dolor (Auyero y Swistun, 2008:38). En este sentido, dentro de la gran variedad de tradiciones de trabajo etnográfico existentes en ciencias sociales, vale la pena recobrar la llamada perspectiva situacional (Agier 1999; Gluckman 1961; Mitchel 1983), la que se configura a partir de dos conceptos clave: la situación y el setting o marco social. La primera se origina en una decisión del investigador, quien busca aislar un evento o un conjunto de eventos para hacer posible una lectura coherente. Esta coherencia es proporcionada, en principio, por una definición cognitiva, vale decir, por la presencia de un mínimo de sentido compartido entre quienes participan de ella. El setting o marco social, por su parte, refiere al conjunto de constricciones globales que pueden determinar algún aspecto de la situación, por ejemplo, el sistema político y administrativo, la organización local del trabajo, la densidad residencial, la homogeneidad o heterogeneidad étnica, etc.

A través de esta perspectiva situacional es posible identificar "casos concretos" para observar la espacialidad relacional de la catástrofe y el sufrimiento de manera "tangible" y "visible". En cada caso seleccionado se debiera prestar atención a los variados actores involucrados, así como a las diferentes escalas sociales y temporales puestas en acción, y a los diversos factores causales operantes. En este último punto, el supuesto general es que entre dichos factores causales hay unos de carácter prioritario o principal, es decir, fenómenos que intervienen como la causa eficiente específica del caso en cuestión: sería el llamado "evento" natural (Cid, et. al., 2012; Aliste y Pérez, 2013; González-Muzzio, 2013; Ugarte y Salgado, 2014), mientras que hay otros factores de carácter secundario, puesto que se asocian a los primeros para dar la sustancia y consistencia específica a las modalidades en que se articula la catástrofe y el sufrimiento: el nivel de vulnerabilidad territorial, pero también las formas de explotación y dominación sociales que afectan a los individuos y los colectivos involucrados en cada caso (Auyero y Swistun, 2008).

La selección de los casos debiera orientarse por la búsqueda de cierta singularidad, de acuerdo a la propuesta de Zussman. Según el autor (Zussman, 2004:362, citado en Auyero, 2007) Los estudios de casos exitosos se concentran en circunstancias extremas, inusuales y en ejemplos analíticamente claros, todos los cuales son importantes no porque sean representativos, sino porque muestran un proceso o un problema en un relieve particularmente claro. En los casos seleccionados interesa la manera en que un "evento" natural interviene las rutinas cotidianas de un conjunto de personas que habitan una localidad. Aquí los focos de atención privilegiados pueden estar puestos en la rutina diaria entendida como "reconquista de la normalidad", la sociabilidad y la colectividad, ya que se pretende observar y describir las formas en que se produce una nueva rutina, particularmente las formas de relación entre individuos y de articulación del colectivo.

El trabajo de campo podría estar organizado en una secuencia de fases que incluirían: i. revisión de prensa y archivos; ii. inmersión inicial en el terreno; iii. entrevistas a actores clave identificados en el trabajo de campo; inmersión sistemática en el terreno; iv. realización de "recorridos comentados" (Thibaud, 2001) con afectados o víctimas; generación de instrumentos de representación visual y cartográfica de las modalidades de articulación socio-espacial identificadas: en particular, mapas mentales (De Alma, 2004; Avry, 2012). 


\section{REFLEXIÓN FINAL}

Finalmente cabría hacer alusión aquí a las orientaciones y sugerencias para el estudio de la sensibilidad de una época que medio siglo atrás planteaba L. Febvre en el texto antes citado (Febvre, 1952). Aunque su foco de atención se encuentra en el análisis historiográfico de las emociones, los planteamientos poseen un carácter programático absolutamente vigente a la luz de las propuestas del "emotional turn" y de la discusión que aquí hemos esbozado. Si hacemos una apretada síntesis de sus consideraciones principales, encontramos que el historiador francés planteaba que la emoción:

- Primero, implica relaciones entre seres humanos (rapports d'homme à homme) y, en consecuencia, relaciones colectivas.

- Segundo, que las emociones llegan a constituir un "sistema de incitaciones interindividuales", por lo que cabe considerarlas como una institución.

- Que las emociones siempre involucran una suerte de ambivalencia, por cuanto ellas incluyen su opuesto.

- Que entre las emociones y su representación existe una suerte de antagonismo, una especie de incompatibilidad, en la medida que las emociones alteran la vida intelectual y que, a su vez, la representación de una emoción es una forma de reprimirla.

- Que los instrumentos para llevar a cabo una historia de la sensibilidad y la vida afectiva son principalmente tres: el vocabulario de una época (a través de su análisis lingüístico y filológico), la iconografía artística y, finalmente, la literatura del período considerado.

- Que el tratamiento de estos materiales informativos debe estar siempre guiado por la precaución, evitando las generalizaciones abusivas.

- Finalmente, que el estudio de las emociones es una empresa colectiva que requiere el concurso de distintas disciplinas.

Colectivas e institucionales. Pero también ambivalentes y de difícil representación. El estudio de las emociones plantea desafíos complejos de abordar incluso en el estado actual de las disciplinas sociales. La investigación sobre catástrofe y el sufrimiento desde una perspectiva relacional instala retos similares. Interdisciplinares, porque tal como plantea Gunaratnam -siguiendo a Bourdieu-, resulta imposible limitar los afectos y efectos del sufrimiento a una sola esfera de la vida y del ser (Gunaratnam 2012:113). Junto con ello, y siguiendo el argumento de Paperman (1992), es posible plantear que el estudio del sufrimiento y la catástrofe es importante porque supone una contribución a la comprensión y configuración del espacio público: la adopción de un punto de vista evaluativo como modo de apreciación pertinente sobre una situación particular. El estudio de Auyero y Swistun (2008) es un buen ejemplo al respecto: al describir el sufrimiento ambiental, los autores configuran una situación e instalan un punto de vista moral para evaluar dicha situación. En este sentido, la investigación sobre las emociones nos recuerda el carácter moral de la realidad en que vivimos. Investigar sobre sufrimiento y catástrofe va en la misma dirección, ya que puede contribuir a mostrar a quienes sufren como sujetos dignos de consideración por parte de los poderes públicos y del conjunto de la sociedad civil.

\section{BIBLIOGRAFÍA}

AHMED, S. (2010) Happy objetcs. En GREGG, M. y SEIGTWORTH, G. (Editores). The Affect Theory Reader. Duke University Press, Durham NC.

ALISTE, E. y PÉREZ, S. (2013) La reconstrucción del Gran Concepción: territorio y catástrofe como permanencia histórica. Revista de geografía Norte Grande, № 54:199-218.

AUYERO, J. y SWISTUN, D. (2008) Inflamable. Estudio del sufrimiento ambiental. Buenos Aires: Editorial Paidós.

AVRY, L. (2012) Analyser les conflits territoriaux par les representations spatiales. Une méthode cognitive par cartes mentales. Tesis doctoral presentada en el UMR CNRS 6590 ESO «Espaces et Société" Laboratoire ESO-Rennes. http://tel.archives-ouvertes.fr/tel-00808779

BACK, L. (2007) The art of listening. Oxford, Berg.

BACK, L. y PUWAR, N. (Editores) (2012) Live methods. London: Blackwell.

BEDARD, M. (2002) Une typologie du haut-lieu ou la quadrature d'un géosymbole. Cahiers de Géographie du Québec, Volume $46, \mathrm{~N}^{\circ}$ 127:49-74.

(2002a) De l'être-ensemble à l'être-au-monde. Le rôle du haut lieu. Ethnologies Musées / Museums, Vol. 24, N²:229-241.

BOLTANSKI, L. (2007) La souffrance à distance: morale humanitaire, médias et politique. Paris: Gallimard.

BOURDIEU, P. (1999) La miseria del Mundo. Buenos Aires: Editorial Fondo de Cultura Económica.

CID-ORTIZ, G., CASTRO CORREA, C. \& RUGIERO DE SOUZA, V. (2012) Percepción del riesgo en relación con capacidades de autoprotección y autogestión, como elementos relevantes en la reducción de la vulnerabilidad en la ciudad de La Serena. Revista INVI, 27(75), 105-142. doi:10.4067/invi. v27i75.565

DAMASIO, A. (2013) El error de Descartes. Buenos Aires: Paidós.

DAVIDSON, J., BONDI, L. y SMITH, M. (2007) Emotional Geographies. London: Ashgate.

DE ALMA, M. (2004) Mapas mentales de la Ciudad de México: una aproximación psicosocial al estudio de las representaciones. Estudios Demográficos y Urbanos 55:115-143.

DUCCI, M. E. (1986) El terremoto en México y las tareas de reconstrucción. ¿Una lección para América Latina? Revista EURE Vol. 13, Núm. 38.

FAVRET-SAADA, J. (1994) "Weber, les émotions et la religion". En Terrain [En ligne], 22 | mars. URL:http://terrain.revues. org/3088; DOI : 10.4000/terrain.3088.

FEBVRE, L. (1992 [1952]) Combats pour l'Histoire. Paris: Armand Colin.

GEERTZ, C. (2002) Cultura, mente, cerebro/cerebro, mente, cultura. Reflexiones antropológicas sobre temas filosóficos. Barcelona: Editorial Paidós. 
GONZÁLEZ-MUZZIO, C. (2013) El rol del lugar y el capital social en la resiliencia comunitaria posdesastre: Aproximaciones mediante un estudio de caso después del terremoto del 27/F. EURE 39(117):25-48.

GUNARATNAM, Y. (2012) Learning to be affected: social suffering and total pain at life's borders. En Back, L. y Puwar, N. (Editores) Live methods. London: Blackwell.

JIRÓN, P. (2010) On Becoming ‘la sombra/the shadow'. En: Büscher, M., Urry, J. y Witchger, K. (Editores) Mobile Methods. London: Routledge.

JIRÓN, P. e ITURRA, L. (2011) Momentos móviles. Los lugares móviles y la nueva construcción del espacio público. Revista Arquitecturas del Sur, $N^{\circ}$ 39:44-57.

KLEINMAN, A., DAS, V. y LOCK, M. (1997) Social Suffering. California: University of California Press.

LAVELL, T. A. (1993) Ciencias Sociales y desastres naturales en América Latina: un encuentro inconcluso. Revista EURE Vol. 19, Núm. 58.

LE BRETON, D. (1999) Antropología del dolor. Barcelona: Seix Barral.

(2002) Antropología del cuerpo y la modernidad. Buenos Aires: Nueva Visión.

MASCAREÑO, A. (2006) Sociología de la felicidad: lo incomunicable. Teología y vida, Vol. XLVII.

MAUSS, M. (1921) L'expression obligatoire des sentiments (rituels oraux funéraires australiens). Journal de psychologie, 18.

MAY, J. y THRIFT, N. (2001) Timespace. London: Routledge.

MOL, A. (2002) The body multiple: ontology in medical practice. Duke University Press.

MORRIS, D. (1991) The culture of pain. California: University of California Press.

MOScoso, J. (2011) Historia cultural del dolor. Madrid: Taurus.

MUSSET, A. (2008) "C'est là que... " Peyruis et les petits hauts lieux d'une mémoire familiale. Cahiers de géographie du Québec, Volume 52, N 145:63-79.

(2010) Vulnerabilidad social, justicia espacial y resiliencia. Concepción, Chile, entre dos terremotos (1751-1835). En: Musset, A. (editor). Ciudad, sociedad, justicia: un enfoque espacial y cultural. Mar del Plata: Eudem, Universidad Nacional de Mar del Plata, 2010, páginas 31-64.

PAPERMAN, P. (1992) Les émotions et l'espace public. Quaderni. N 18, Automne 1992. Les espaces publics. Páginas 93-107.

POREE, J. (1997) Souffrance et temps. Esquisse phénoménologique. Revue Philosophique de Louvain. Quatrième série, Tome 95, $\mathrm{N}^{\circ}$ 1:103-129.

REVEL, J. (2005) Un momento historiográfico: Trece ensayos de historia social. Buenos Aires: Manantial.

ROSALDO, M. (1980) Knowledge and passion. Cambridge: Cambridge University Press.

ROSALDO, R. (1989) Cultura y Verdad. Nueva propuesta de análisis social. México: Grijalbo.
SMITH, M., DAVIDSON, J., CAMERON, L. y BONDI, L. (2009) Emotion, place and culture. London: Ashgate.

THRIFT, N. (2006) Space. Theory Culture Society, N²3:139-146.

(2008) I Just Don't Know What Got Into Me: Where Is the Subject? Subjectivity, $N^{\circ} 22: 82-89$.

THUMALA, D. (2003) Modernidad y Sufrimiento: Algunos elementos para la comprensión de la significación. Revista Mad N 8, Mayo 2003. Departamento de Antropología, Universidad de Chile.

UGARTE, A. y SALGADO, M. (2014) Sujetos en emergencia: acciones colectivas de resistencia y enfrentamiento del riesgo ante desastres; el caso de Chaitén, Chile. Revista INVI, 29(80):143-168.

WIERZBICKA, A. y JAMROZIK, E. (1988) L'amour, la colère, la joie, l'ennui. La sémantique des émotions dans une perspective transculturelle. Langages, 23e année, N 89 :97-107.

WILKINSON, I. (2005) Suffering: A Sociological Introduction. Cambridge: Polity Press.

WITTGENSTEIN, L. (2008) Investigaciones filosóficas. Barcelona: Crítica.

ZOMBORY-NAGY, P. y FRANDON, V. (1994) Pour une histoire de la souffrance: expressions, représentations, usages. Médiévales, $\mathrm{N}^{\circ} 27: 5-14$.

\section{NOTAS}

1 En la aproximación de Thrift (2006) al espacio hay cinco postulados que resultan fundamentales: i. todo está espacialmente distribuido; ii. los espacios son porosos, por lo que se hace difícil establecer fronteras; iii. todo espacio está en constante movimiento; iv. no hay un solo tipo de espacio y; v. no hay espacio separado del tiempo (timespace). Estos planteamientos tienen importantes implicancias en las formas convencionales de entender la causalidad y la eficacia (Thrift, 2006:145), especialmente en lo que refiere a las modalidades de estar con otros, de afectarlos, así como en las formas de organizarnos mutuamente.

2 Cabe aquí señalar que una fuente de inspiración metodológica ha sido la propuesta de live sociology de L. Back, la que se opone, punto por punto, a lo que él denomina dead sociology, que se encuentra en retirada, pero que ha sido dominante hasta nuestros días. Para el autor, la dead sociology posee cuatro características centrales, entre las que quisiera destacar las dos iniciales: primero, es objetificante, es decir, propone objetos inmóviles y "muertos" para dar cuenta de cosas vivas y bullentes. Segundo, es confortable, pues se queda en una perspectiva a priori que evita tomar en consideración cuestiones desconcertantes, desconocidas, etc., propias del trabajo empírico.

3 La precaución sobre el nivel discursivo debe ser bien entendida. Se trata de un nivel imprescindible, pero que debe ser complementado con otros, eminentemente prácticos.

56 > Revista Márgenes № 15 Vol 11 > Diciembre 2014: 49 a 56 Facultad de Arquitectura > Universidad de Valparaíso 